\title{
THE FUNDAMENTAL GROUP OF CERTAIN DELETED PRODUCT SPACES
}

\author{
BY \\ C. W. PATTY(1)
}

1. Introduction. The deleted product space $X^{*}$ of a topological space $X$ is the subset of the cartesian product of $X$ with itself consisting of pairs of distinct points. The computation of the homotopy and homology groups of deleted product spaces is one step toward the topological classification of spaces by means of algebraic invariants. The usefulness of these groups in distinguishing spaces of the same homotopy type has been illustrated by $\mathrm{Hu}$ [3]. However there are nonhomeomorphic spaces $A$ and $B$ such that $A^{*}$ and $B^{*}$ have the same homotopy type.

In the present paper, we undertake the computation of $\pi_{1}\left(X^{*}\right)$ and $H_{k}\left(X^{*}, Z\right)$, where $X$ is a connected, finite, 1-dimensional polyhedron, $Z$ is the group of integers, and $k=1,2$. To do this we note that (see Remark 2.1) a homeomorph of every such polyhedron $X$, which is not an arc, can be constructed by adding 1-simplexes either to a simple closed curve or a triod. It can be arranged so that each step is one of two kinds: (1) the addition of a 1-simplex incident to one vertex, or (2) the addition of a 1-simplex in such a way that it is then incident to two vertices of order 2. Theorems are proved which show that: (1) If $X$ is obtained from $A$ by addition of the first kind, then $\pi_{1}\left(X^{*}\right)$ is the free product of $\pi_{1}\left(A^{*}\right)$ and the free group on $2 n-4$ generators, where $n$ is the order in $X$ of the incident vertex (see Theorem 3.1). (2) If $X$ is obtained from $A$ by addition of the first kind, then $H_{2}\left(X^{*}, Z\right)$ is isomorphic to $H_{2}\left(A^{*}, Z\right)$ (see Theorem 3.2). (3) If $X$ is obtained from $A$ by addition of the second kind and if there is a presentation $\left(x_{1}, \ldots, x_{p} / r_{1}, \ldots, r_{q}\right)$ of $\pi_{1}\left(A^{*}\right)$, where each $r_{i}$ is a commutativity relation between elements, then there is a presentation $\left(y_{1}, \ldots, y_{p+2-2 n} / s_{1}, \ldots, s_{q+2 x-2 n}\right)$ of $\pi_{1}\left(X^{*}\right)$, where each $s_{i}$ is a commutativity relation between elements, $\alpha$ is the integer such that $\pi_{1}(A)$ is the free group on $\alpha$ generators, and $n$ is the deleted product number (see Definition 4.4) of $A$ with respect to the two incident vertices (see Theorem 4.1). (4) If $X$ is obtained from $A$ by addition of the second kind, then $H_{2}\left(X^{*}, Z\right)$ is the direct product of $H_{2}\left(A^{*}, Z\right)$ and the free abelian group on $2 \alpha-2 n$ generators (see Theorem 4.2). An important fact about this deleted product number is that it is obtained by considering only the structure of $A$.

Presented to the Society, November 18, 1960; received by the editors October 31, 1960 and, in revised form, October 20, 1961.

(1) This work was supported by the University of Georgia Alumni Foundation, the Research Corporation, and the National Science Foundation under Grant NSF G-9418. 
Briefly, an outline of the results is as follows. In $\$ 2$, we note that there is a deformation retraction of the deleted product space onto a certain polyhedron, and we compute the fundamental group of the deleted product space of: (1) a triod, and (2)a simple closed curve. We also note that the deleted product space of a connected, finite, 1-dimensional polyhedron is arcwise connected if the polyhedron is not an arc.

In $\S 3$ and 4, we prove the formulas listed above for additions of the first and second kind, respectively. It is an immediate consequence of these results that if $X$ is a connected, finite, 1-dimensional polyhedron, then $H_{1}\left(X^{*}, Z\right)$ is a free abelian group, and the number of generators of this group is obtained immediately from the presentation of $\pi_{1}\left(X^{*}\right)$. Note that if $\left(x_{1}, \ldots, x_{p} / r_{1}, \ldots, r_{q}\right)$ is a presentation of $\pi_{1}\left(X^{*}\right)$, where each $r_{i}$ is a commutativety relation between elements, then $H_{1}\left(X^{*}, Z\right)$ is the free abelian group on $p$ generators and $H_{2}\left(X^{*}, Z\right)$ is the free abelian group on $q$ generators.

The author wishes to express his gratitude to Professors T. R. Brahana and M. L. Curtis.

2. Some preliminary theorems. Definition 2.1. If $X$ is a topological space, then the diagonal, $D_{X}$, of $X \times X$ is the set of all points of the form $(x, x)$, where $x \in X$.

Definition 2.2. If $X$ is a topological space, then the deleted product space, $X^{*}$, of $X$ is the space $X \times X-D_{X}$ with the relative topology.

Definition 2.3. If $X$ is a connected, finite, 1-dimensional polyhedron and $A$ and $B$ are subpolyhedra of $X$, let $P\left(A \times B-D_{X}\right)=\bigcup\{r \times s \mid r$ is a simplex of $A$, $s$ is a simplex of $B$, and $r \cap s=\varnothing\}$. Let $\sigma$ be a cell of $P\left(A \times B-D_{X}\right)$ if and only if $\sigma=r \times s$, where $r$ is a simplex of $A$, $s$ is a simplex of $B$, and $r \cap s=\emptyset$.

The following theorem is proved by Shapiro (see [4, p. 257]).

THEOREM 2.1. If $X$ is a connected, finite, 1-dimensional polyhedron, then $P\left(X^{*}\right)$ is a polyhedron and there is a deformation retraction of $X^{*}$ onto $P\left(X^{*}\right)$.

If $X$ is an arc, then the deleted product space of $X$ consists of two components each of which is contractible to a point. Therefore in all of our theorems we assume that $X$ is not an arc.

THEOREM 2.2. If $X$ is a triod, then $P\left(X^{*}\right)$ is a simple closed curve and hence $\pi_{1}\left(X^{*}\right)$ is infinite cyclic.

Proof. By Theorem 2.1, there is a deformation retraction of $X^{*}$ onto $P\left(X^{*}\right)$. The polyhedron $P\left(X^{*}\right)$ is a simple closed curve. Therefore $\pi_{1}\left(X^{*}\right)$ is infinite cyclic.

LeMma 2.1. If $X$ and $Y$ are connected, finite, 1-dimensional polyhedra such that $X$ is homeomorphic to $Y$, then $X^{*}$ is homeomorphic to $Y^{*}$.

Proof. Obvious. 
THEOREM 2.3. If $X$ is a simple closed curve, then $X^{*}$ has the homotopy type of a simple closed curve. Hence $\pi_{1}\left(X^{*}\right)$ is infinite cyclic.

Proof. The simple closed curve $X$ is homeomorphic to the boundary $Y$ of a 2-simplex. Therefore, by Lemma 2.1, $X^{*}$ is homeomorphic to $Y^{*}$. By Theorem 2.1 , there is a deformation retraction of $Y^{*}$ onto $P\left(Y^{*}\right)$. The polyhedron $P\left(Y^{*}\right)$ is a simple closed curve.

THEOREM 2.4. If $X$ is a connected, finite, 1-dimensional polyhedron which is not an arc, then $X^{*}$ is arcwise connected.

The above theorem is an immediate consequence of a theorem of Eilenberg (see $[1$, p. 43]).

REMARK 2.1. Let $X$ be a connected, finite, 1-dimensional polyhedron which is not an arc. If $X$ does not have a vertex of order $\geqq 3$, then $X$ is a simple closed curve. If $X$ does have a vertex of order $\geqq 3$, let $A^{\prime}$ be a triod in $X$. Then it is clear that there is a subdivision $X^{\prime}$ of $X$ such that: (1) each simplex of $A^{\prime}$ is a simplex of $X^{\prime}$, (2) $X^{\prime}$ consists of a finite number of 1 -simplexes, $r_{1}, \ldots, r_{k}$, and (3) $X^{\prime}$ can be realized by starting with $A^{\prime}$ and adding one 1-simplex $r_{j}$ at a time so that either $r_{j} \cap\left(\bigcup_{k=1}^{j-1} r_{k}\right)$ is a single vertex or $r_{j} \cap\left(\bigcup_{k=1}^{j-1} r_{k}\right)$ consists of two vertices $v_{1}, v_{2}$, where each $v_{1}$ is a vertex of order 1 in $\bigcup_{k=1}^{j-1} r_{k}$. In this paper, we shall assume that such a subdivision of $X$ has been made.

REMARK 2.2. Let $A$ and $B$ be finite, 1-dimensional polyhedra such that $A \cap B$ $=\{p\}$, where $p$ is a vertex of $A$ and $B$. Let $X=A \cup B$. Then we have the following relations:

(1) $X^{*}=A^{*} \cup B^{*} \cup[(A \times B)-\{(p, p)\}] \cup[(B \times A)-\{(p, p)\}]$,

(2) $A^{*} \cap B^{*}=\varnothing$,

(3) $[(A \times B)-\{(p, p)\}] \cap[(B \times A-\{(p, p)\}]=\varnothing$,

(4) $A^{*} \cap[(A \times B)-\{(p, p)\}]=[(A \times p)-\{(p, p)\}]$,

(5) $A^{*} \cap[(B \times A)-\{(p, p)\}]=[(p \times A)-\{(p, p)\}]$,

(6) $B^{*} \cap[(A \times B)-\{(p, p)\}]=[(p \times B)-\{(p, p)\}]$,

(7) $B^{*} \cap[(B \times A)-\{(p, p)\}]=[(B \times p)-\{(p, p)\}]$.

REMARK 2.3. Let $A$ and $B$ be finite, 1-dimensional polyhedra such that $A \cap B=\{x\} \cup\{y\}$, where $x$ and $y$ are vertices of $A$ and $B$. Let $X=A \cup B$. Then we have the following relations:

(1) $X^{*}=A^{*} \cup B^{*} \cup[(A \times B)-(\{(x, x)\} \cup\{(y, y)\})]$

$$
\cup[(B \times A)-(\{(x, x)\} \cup\{(y, y)\})],
$$

(2) $A^{*} \cap B^{*}=\{(x, y)\} \cup\{(y, x)\}$,

(3) $[(A \times B)-(\{(x, x)\} \cup\{(y, y)\})]$

$\cap[(B \times A)-(\{(x, x)\} \cup\{(y, y)\})]=\{(x, y)\} \cup\{(y, x)\}$,

(4) $A^{*} \cap[(A \times B)-(\{(x, x)\} \cup\{(y, y)\})]$

$$
=[(A \times x)-\{(x, x)\}] \cup[(A \times y)-\{(y, y)\}],
$$


(5) $A^{*} \cap[(B \times A)-(\{(x, x)\} \cup\{(y, y)\})]$

(6) $B^{*} \cap[(A \times B)-(\{(x, x)\} \cup\{(y, y)\})]$

$$
=[(x \times A)-\{(x, x)\}] \cup[(y \times A)-\{(y, y)\}] \text {, }
$$$$
=[(x \times B)-\{(x, x)\}] \cup[(y \times B)-\{(y, y)\}],
$$

(7) $B^{*} \cap[(B \times A)-(\{(x, x)\} \cup\{(y, y)\})]$

$$
=[(B \times x)-\{(x, x)\}] \cup[(B \times y)-\{(y, y)\}] \text {. }
$$

\section{Addition of a 1-simplex at one vertex}

THEOREM 3.1. Let $A$ be a connected, finite, 1-dimensional polyhedron which is not an arc, and let $B$ be a 1-simplex. If $X=A \cup B$ and $A \cap B=\left\{v_{0}\right\}$, where $v_{0}$ is a vertex of order $n$ in $X$, then $\pi_{1}\left(X^{*}\right)$ is the free product of $\pi_{1}\left(A^{*}\right)$ and the free group on $2 n-4$ generators.

Proof. By Theorem 2.1, there is a deformation retraction of $X^{*}$ onto $P\left(X^{*}\right)$. Thus we want to show that $\pi_{1}\left(P\left(X^{*}\right)\right)$ is the free product of $\pi_{1}\left(P\left(A^{*}\right)\right)$ and the free group on $2 n-4$ generators. It follows from Remark 2.2, part (1), and Definition 2.3 that

$$
P\left(X^{*}\right)=P\left(A^{*}\right) \cup P\left(B \times A-D_{X}\right) \cup P\left(A \times B-D_{X}\right) .
$$

First we show that $\pi_{1}\left(P\left(A^{*}\right) \cup P\left(B \times A-D_{X}\right)\right)$ is the free product of $\pi_{1}\left(P\left(A^{*}\right)\right)$ and the free group on $n-2$ generators. It is clear that $P\left(A^{*}\right) \cup P\left(B \times A-D_{X}\right)$ is arcwise connected since $(B \times A) \cap D_{X}=\left\{\left(v_{0}, v_{0}\right)\right\}$ and

$$
P\left(A^{*}\right) \cap P\left(B \times A-D_{X}\right) \neq \varnothing .
$$

Let $b$ be the other vertex of $B$, let $v_{i}(i=1,2, \ldots, n-1)$ be a vertex of a 1 simplex of $A$ which has $v_{0}$ as its other vertex, and let $v_{i}(i=n, n+1, \ldots, k)$ be the remaining vertices of $A$. To obtain $P\left(A^{*}\right) \cup P\left(B \times A-D_{X}\right)$ from $P\left(A^{*}\right)$, the following cells are added:

$$
\begin{aligned}
& \text { 2-cells } B \times\left[v_{i}, v_{j}\right], \quad i, j \neq 0, \\
& \text { 1-cells } \begin{cases}B \times v_{i}, & i \neq 0, \\
b \times\left[v_{i}, v_{j}\right], & \text { all } i, j,\end{cases} \\
& 0 \text {-cells } \quad b \times v_{i}, \quad \text { all } i \text {. }
\end{aligned}
$$

Suppose that an auxiliary path system is given in $P\left(A^{*}\right)$, and extend this system to include the new vertices as follows:

If $i \neq 0$ and $h_{i}$ is the auxiliary path to $\left(v_{0}, v_{i}\right)$, put $h_{i}$ followed by $B \times v_{i}$ as the auxiliary path to $\left(b, v_{i}\right)$.

Let the auxiliary path to $\left(b, v_{0}\right)$ be $h_{1}$ followed by $B \times v_{1}$ and $b \times\left[v_{1}, v_{0}\right]$.

The remaining new 1-cells $b \times\left[v_{i}, v_{j}\right]\left(b \times\left[v_{0}, v_{1}\right]\right.$ excepted $)$ are associated with new generators of the edge path group. However, the new generators associated with $b \times\left[v_{i}, v_{j}\right], i, j \neq 0$, are killed by the relations associated with the new 2-cells $B \times\left[v_{i}, v_{j}\right], i, j \neq 0$. The remaining $n-2$ generators, $b \times\left[v_{0}, v_{i}\right], i=2,3, \ldots, n-1$, are just added to the presentation of the edge path group of $P\left(A^{*}\right)$, so that 


$$
\pi_{1}\left(P\left(A^{*}\right) \cup P\left(B \times A-D_{X}\right)\right)
$$

is the free product of $\pi_{1}\left(P\left(A^{*}\right)\right)$ and the free group on $n-2$ generators.

By a similar argument, it can be shown that $\pi_{1}\left(P\left(X^{*}\right)\right)$ is the free product of $\pi_{1}\left(P\left(A^{*}\right) \cup P\left(B \times A-D_{X}\right)\right)$ and the free group on $n-2$ generators, which completes the proof of the theorem.

THEOREM 3.2. Let $A$ be a connected, finite, 1-dimensional polyhedron which is not an arc, and let $B$ be a 1-simplex. If $X=A \cup B$ and $A \cap B=\left\{v_{0}\right\}$, where $v_{0}$ is a vertex in $X$, then $\mathrm{H}_{2}\left(X^{*}, \mathrm{Z}\right)$ is isomorphic to $\mathrm{H}_{2}\left(A^{*}, \mathrm{Z}\right)$.

Proof. If $C$ is a 2-cycle of $P\left(X^{*}\right)$, then it is easy to see that every 2-simplex of $C$ with nonzero coefficient is a 2-simplex of $P\left(A^{*}\right)$. Therefore $Z_{2}\left(P\left(A^{*}\right), Z\right)$ is isomorphic to $Z_{2}\left(P\left(X^{*}\right), Z\right)$, and hence $H_{2}\left(P\left(A^{*}\right), Z\right)$ is isomorphic to $H_{2}\left(P\left(X^{*}\right), Z\right)$.

\section{Addition of a 1-simplex at both vertices.}

Notation. Let $X$ be a finite, 1-dimensional polyhedron, and let $C_{1}\left(X, Z_{2}\right)$ denote the group of 1-chains of $X$ with coefficients in $Z_{2}$, the integers mod 2. If $S$ is a simple closed curve in $X$, let $r_{1}, \ldots, r_{n}$ be the 1 -simplexes of $S$. Then there is associated with $S$ a unique element $S=\sum_{i=1}^{n} r_{i} \in C_{1}\left(X, Z_{2}\right)$, and $S$ is called a 1-circuit.

DEFINITION 4.1. In a finite, 1-dimensional polyhedron, a simple closed curve $S$ is a linear combination of simple closed curves $S_{1}, S_{2}, \ldots, S_{k}$ if and only if for the associated chains in $C_{1}\left(X, Z_{2}\right), \hat{S}=a_{1} \hat{S}_{1}+a_{2} \hat{S}_{2}+\ldots+a_{k} \hat{S}_{k}$. (Thus the operation + is chain addition, and the coefficients $a_{i} \in Z_{2}$. Observe that the sum of 1-circuits may not be a 1-circuit. However, in this definition, we consider only those sums of 1-circuits which are 1-circuits.)

Definition 4.2. The simple closed curves $S_{1}, \ldots, S_{k}$ in a finite, 1-dimensional polyhedron $X$ are linearly dependent if and only if some one of the simple closed curves $S_{i}$ is a linear combination of the preceding ones. Simple closed curves which are not linearly dependent are called linearly independent.

Definition 4.3. A basis for the simple closed curves in a finite, 1-dimensional polyhedron $X$ is a linearly independent set $C$ of simple closed curves such that any simple closed curve in $X$ is a linear combination of members of $C$.

Proposition 4.1. If $X$ is a finite, 1-dimensional polyhedron and $S_{1}, S_{2}, \ldots, S_{\alpha}$ is a basis for the simple closed curves in $X$, then $\left[\hat{S}_{1}\right],\left[\hat{S}_{2}\right], \ldots,\left[\hat{S}_{\alpha}\right]$ is a basis for $H_{1}\left(X, Z_{2}\right)$.

Proof. The proof is an immediate consequence of the fact that every 1-cycle in $X$ is a linear combination of 1-circuits.

COROLlaRY 4.1. If $X$ is a finite, 1-dimensional polyhedron, then any two bases for the simple closed curves in $X$ have the same number of elements. 
Definition 4.4. Let $A$ be a connected, finite, 1-dimensional polyhedron which is not an arc, and let $x$ and $y$ be vertices of order 1 . We define a number $n$ called the deleted product number of $A$ with respect to $x$ and $y$ as follows:

For every finite collection $G$ of simple closed curves in $A$, let $n(G)$ denote the number of elements of $G$ which separate $x$ from $y$ in $A$. Let

$$
n=\min \{n(G) \mid G \text { is a basis for a simple closed curves in } A\} .
$$

TheOREM 4.1. Let $X=A \cup B$, where $A$ is a connected, finite, 1-dimensional polyhedron which is not an arc and $B$ is a 1-simplex. Suppose $A \cap B=\left\{v_{1}\right\}$ $\cup\left\{v_{2}\right\}$, where $v_{1}$ and $v_{2}$ are vertices of order 2 in $X$. Let $n$ be the deleted product number of $A$ with respect to $v_{1}$ and $v_{2}$, and let $\alpha$ be the number of elements in a basis for the simple closed curves in $A$. If $\left(x_{1}, \ldots, x_{p} / r_{1}, \ldots, r_{q}\right)$ is a presentation of $\pi_{1}\left(A^{*}\right)$, where each $r_{i}$ is a commutativity relation between elements, then there is a presentation $\left(y_{1}, \ldots, y_{p+2-2 n} / s_{1}, \ldots, s_{q+2 \alpha-2 n}\right)$ of $\pi_{1}\left(X^{*}\right)$, where each $s_{i}$ is a commutativity relation between elements.

Proof. By Theorem 2.1, $\pi_{1}\left(P\left(A^{*}\right)\right)$ is isomorphic to $\pi_{1}\left(A^{*}\right)$ and $\pi_{1}\left(P\left(X^{*}\right)\right)$ is isomorphic to $\pi_{1}\left(X^{*}\right)$. It follows from Remark 2.3 and Definition 2.3 that

$$
P\left(X^{*}\right)=P\left(A^{*}\right) \cup P\left(B \times A-D_{X}\right) \cup P\left(A \times B-D_{X}\right)
$$

and

$$
P\left(A^{*}\right) \cap P\left(B \times A-D_{X}\right)=P\left(v_{1} \times A-D_{X}\right) \cup P\left(v_{2} \times A-D_{X}\right) .
$$

Observe that $P\left(A^{*}\right) \cup P\left(B \times A-D_{X}\right)=P\left(X \times A-D_{X}\right)$. First we show that there is a presentation $\left(\gamma_{1}, \ldots, \gamma_{p+1-n} / \delta_{1}, \ldots, \delta_{q+\alpha-n}\right)$ of $\pi_{1}\left(P\left(X \times A-D_{X}\right)\right)$, where each $\delta_{i}$ is a commutativity relation between elements.

Let $u_{1}, u_{2}, \ldots, u_{k}$ denote the vertices of $A$ different from $v_{1}$ and $v_{2}$. To obtain $P\left(X \times A-D_{X}\right)$ from $P\left(A^{*}\right)$, the following cells are added:

$$
\begin{aligned}
& \text { 2-cells } B \times\left[u_{i}, u_{j}\right], \quad \text { all } i, j, \\
& 1 \text {-cells } B \times u_{i}, \quad \text { all } i .
\end{aligned}
$$

Let $G=\left\{S_{1}, S_{2}, \ldots, S_{\alpha}\right\}$ be a basis for the simple closed curves in $A$ chosen so that the number of elements of $G$ which separate $v_{1}$ from $v_{2}$ in $A$ is $n$.

The inclusion map $k_{1}: P\left(v_{1} \times A-D_{A}\right) \rightarrow P\left(A^{*}\right)$ induces an isomorphism of the edge path group of $P\left(v_{1} \times A-D_{A}\right)$ into the edge path group of $P\left(A^{*}\right)$, and we may assume that $x_{1}, x_{2}, \ldots, x_{\alpha}$ are represented by 1 -cells of the form $v_{1} \times \sigma_{1}$, $v_{1} \times \sigma_{2}, \ldots, v_{1} \times \sigma_{\alpha}$, where $\sigma_{j}$ is a 1-simplex of $S_{j}$ for each $j=1,2, \ldots, \alpha$.

Also the inclusion map $k_{2}: P\left(v_{2} \times A-D_{A}\right) \rightarrow P\left(A^{*}\right)$ induces an isomorphism of the edge path group of $P\left(v_{2} \times A-D_{A}\right)$ into the edge path group of $P\left(A^{*}\right)$, and $v_{1} \times \sigma_{j}$ and $v_{2} \times \sigma_{j}$ represent the same element of the edge path group of $P\left(A^{*}\right)$ if and only if there is a path in $A$ from $v_{1}$ to $v_{2}$ which does not intersect $S_{j}$.

Suppose $S^{\prime}$ and $S^{\prime \prime}$ are such that every arc in $A$ from $v_{1}$ to $v_{2}$ intersects both $S^{\prime}$ and $S^{\prime \prime}$ and $S=S^{\prime}+S^{\prime \prime}$ is a simple closed curve. If there is an arc in A from $v_{1}$ 
to $v_{2}$ which does not intersect $S$, then $v_{1} \times \sigma$ and $v_{2} \times \sigma$, where $\sigma$ is a 1-simplex of $S$, represent the same element of the edge path group. Therefore $v_{1} \times \sigma^{\prime}, v_{1} \times \sigma^{\prime \prime}$, $v_{2} \times \sigma^{\prime}$, and $v_{2} \times \sigma^{\prime \prime}$ cannot represent four elements of the set of generators of the edge path group of $P\left(A^{*}\right)$ if the only relations in the presentation are commutativity relations. However if every arc from $v_{1}$ to $v_{2}$ intersects $S$, then we may assume that $v_{1} \times \sigma^{\prime}, v_{1} \times \sigma^{\prime \prime}, v_{2} \times \sigma^{\prime}$ and $v_{2} \times \sigma^{\prime \prime}$ represent four elements of the set of generators even though the only relations are commutativity relations. Now it is not possible to obtain a basis $G^{\prime}$ for the simple closed curves in $A$ from the basis $G$ by replacing a simple closed curve $S_{j} \in G$ which is such that every arc from $v_{1}$ to $v_{2}$ intersects $S_{j}$ by a simple closed curve $S_{j}^{\prime}$ which is such that there is an arc from $v_{1}$ to $v_{2}$ which does not intersect $S_{j}^{\prime}$.

Therefore we may assume that an auxiliary path system is given in $P\left(A^{*}\right)$ so that: (1) the 1-cells $v_{1} \times \sigma_{1}, v_{1} \times \sigma_{2}, \ldots, v_{1} \times \sigma_{\alpha}$ represent $\alpha$ elements of the set $x_{1}, x_{2}, \ldots, x_{p}$, and (2) if $S_{1}, S_{2}, \ldots, S_{n}$ are the elements of $G$ which separates $v_{1}$ from $v_{2}$ in $A$, then the 1-cells $v_{2} \times \sigma_{1}, v_{2} \times \sigma_{2}, \ldots, v_{2} \times \sigma_{n}$ represent $n$ elements of the set $x_{1}, x_{2}, \ldots, x_{p}$.

Since every vertex of $P\left(X \times A-D_{X}\right)$ is a vertex of $P\left(A^{*}\right)$, the auxiliary path system in $P\left(A^{*}\right)$ is an auxiliary path system in $P\left(X \times A-D_{X}\right)$.

The new 1-cells are associated with new generators of the edge path group. However all of these new generators, except one, are killed by the relations associated with the new 2-cells. Also if $S_{j}$ separates $v_{1}$ from $v_{2}$ in $A$, then the relations associated with the new 2-cells in $B \times S_{j}$ kill the generator $v_{2} \times \sigma_{j}$. Therefore there are $n$ generators of the edge path group of $P\left(A^{*}\right)$ which are killed by these relations. However if $S_{j}$ does not separate $v_{1}$ from $v_{2}$ in $A$, then the relations associated with the new 2-cells in $B \times S_{j}$ add a commutativity relation to the presentation of the edge path group.

Now to complete the proof of the theorem, we consider

$$
P\left(X \times A-D_{X}\right) \cup P\left(A \times B-D_{X}\right)=P\left(X^{*}\right)
$$

and essentially repeat the above argument.

TheOREM 4.2. Let $X=A \cup B$, where $A$ is a connected, finite, 1-dimensional polyhedron which is not an arc and $B$ is a 1-simplex. Suppose $A \cap B=\left\{v_{1}\right\}$ $\cup\left\{v_{2}\right\}$, where $v_{1}$ and $v_{2}$ are vertices of order 2 in $X$. Let $\alpha$ be the number of elements in a basis for the simple closed curves in $A$, and let $n$ be the deleted product number of $A$ with respect to $v_{1}$ and $v_{2}$. Then $\mathrm{H}_{2}\left(X^{*}, Z\right)$ is the direct product of $\mathrm{H}_{2}\left(A^{*}, Z\right)$ and the free abelian group on $2 \alpha-2 n$ generators.

Proof. Now $\left(P\left(X \times A-D_{X}\right) ; P\left(A^{*}\right), P\left(B \times A-D_{X}\right)\right)$ is a proper triad (see $[2, \mathrm{p} .76])$, and it follows from the Mayer-Vietoris sequence $([2, \mathrm{p} .39])$ of this proper triad that $H_{2}\left(P\left(X \times A-D_{X}\right), Z\right)$ is the direct product of $\mathrm{H}_{2}\left(P\left(A^{*}\right), Z\right)$ and the free abelian group on $\alpha-n$ generators. Then the theorem follows from the 
Mayer-Vietoris sequence or the proper triad

$$
\left(P\left(X^{*}\right) ; P\left(X \times A-D_{X}\right), P\left(A \times B-D_{X}\right)\right) .
$$

\section{BIBLIOGRAPHY}

1. S. Eilenberg, Ordered topological spaces, Amer J. Math. 63 (1941), 39-45.

2. S. Eilenberg and N. Steenrod, Foundations of algebraic topology, Princeton Univ. Press, Princeton, N.J., 1952.

3. S. T. Hu, Isotopy invariants of topological spaces, Proc. Roy. Soc. 255 (1960), 331-366.

4. Arnold Shapiro, Obstructions to the imbedding of a complex in a euclidean space. I. The first obstruction, Ann. of Math. (2) 66 (1957), 256-269.

UNIVERSITY OF GEORGIA, Athens, Georgia and

University of North Carolina,

Chapel Hill, North Carolina 